\title{
Extração e Recomendação de Boas e Más Práticas Pedagógicas a Partir de Processos de Ensino e Aprendizagem usando um Sistema Tutor Inteligente Gamificado
}

\author{
Sivaldo Joaquim de Santana ${ }^{1}$, Ig Ibert Bittencourt ${ }^{1}$, Rafael de Amorim Silva ${ }^{1}$, \\ Patrícia Leone Espinheira ${ }^{2}$
}

${ }^{1}$ Núcleo de Excelência em Tecnologias Sociais (NEES) - Instituto de Computação (IC) Universidade Federal de Alagoas (UFAL) - CEP: 57.072-900 - Maceió, AL - Brasil.

${ }^{2}$ Centro de Ciências Exatas e da Natureza (CCEN) - Departamento de Estatística (DE) Universidade Federal de Pernambuco (UFPE) - Recife, PE - Brasil.

\{sivaldojoaquim, ig.ibert, rafael\}@ic.ufal.br, patespipa@de.ufpe.br

\begin{abstract}
Resumo. Recentemente, alguns indicadores educacionais revelaram que uma parcela significativa dos estudantes da Educação Básica apresenta domínio insuficiente em leitura (Língua Portuguesa) e na resolução de problemas (Matemática). Entretanto, existe uma grande expectativa de que o acesso às tecnologias de aprendizagem adaptativa auxilie na prática docente e contribua para melhoria da aprendizagem. Este artigo tem como objetivo extrair boas e más práticas pedagógicas a partir de processos de ensino e de aprendizagem usando um Sistema Tutor Inteligente (STI) gamificado no Ensino Fundamental. Para tanto, realizou-se um estudo misto com design de experimento controlado com professores e estudantes. Os principais resultados mostram evidências significativas de melhoria na aprendizagem dos estudantes no domínio de Língua Portuguesa e Matemática e apresenta 10 boas práticas e 9 más práticas pedagógicas com o uso de STI gamificado.
\end{abstract}

\begin{abstract}
Recently, some indicators have revealed that a significant part of students from basic education present insufficient domain on reading (Portuguese Language) and on problem resolution (Mathematics). However, there is a large expectation from that the access to adaptive learning technologies aid in teaching practice and to contribute to improved learning. This work aims to extract good and bad pedagogical practices from teaching and learning processes using a Gamified Intelligent Tutor System (ITS) in the Elementary School. We performed a mixed empirical study with controlled experimental design with teachers and students. The main results show significant evidence of improvement in students learning in Portuguese and Mathematics and presents 10 good practices and 9 bad pedagogical practices with the usage of gamified ITS.
\end{abstract}

\section{Introdução}

No Brasil, apesar do avanço ao acesso à Educação Básica da população de 6 a 14 anos [Brasil 2018], ainda existe um percentual significativo de estudantes nessa faixa etária com baixo nível de proficiência em leitura e interpretação de textos (Língua Portuguesa) 
VII Congresso Brasileiro de Informática na Educação (CBIE 2018)

Anais do XXIX Simpósio Brasileiro de Informática na Educação (SBIE 2018)

e na resolução de problemas (Matemática) [OECD 2016; Brasil 2016]. Segundo os resultados da Prova Brasil 2015 realizado pelo Instituto Nacional de Estudos e Pesquisas Educacionais Anísio Teixeira (INEP) do Ministério da Educação (MEC), apenas $51 \%$ dos estudantes aprenderam o adequado em Língua Portuguesa e 39\% em Matemática, até o $5^{\circ}$ ano do Ensino Fundamental I. Para os estudantes do $9^{\circ}$ ano do Ensino Fundamental II, essa proporção reduz para 29\% e 13\%, respectivamente [QEdu 2018]. Além disso, o desempenho dos estudantes brasileiros no PISA $^{1} 2015$ ficou abaixo da média dos países membros da Organização para a Cooperação e Desenvolvimento Econômico (OCDE) em leitura (407 pontos), matemática (377 pontos) e ciências (401 pontos) comparadas às médias da OCDE de 493, 490 e 493 pontos, respectivamente. [OECD 2016; Brasil 2016].

Esses indicadores educacionais revelaram que uma parcela significativa dos estudantes da Educação Básica apresenta domínio insuficiente em Língua Portuguesa e Matemática, áreas cognitivas essenciais para prosseguir na vida acadêmica, para o exercício da cidadania e sua qualificação profissional. Entretanto, existe uma grande expectativa de que o acesso às tecnologias educacionais de aprendizagem adaptativa $\mathrm{e}$ sua adoção na escola seja uma ferramenta potencial para auxiliar o professor e impactar de forma significativa os resultados pedagógicos [Barbosa 2016]. Segundo [de Santana et al. 2016] uma das tecnologias educacionais mais promissoras e eficazes de adaptação são os Sistemas Tutores Inteligentes (STI). Para [Steenbergen-Hu e Cooper 2014] os STI são ambientes de aprendizagem assistido por computadores construídos com base em modelos computacionais desenvolvidos nas ciências da aprendizagem, cognitivas, matemática e inteligência artificial. A aplicação de STI no contexto educacional pode auxiliar nos processos de ensino e aprendizagem e permitir uma maior integração entre estudantes e professores, independente da localização geográfica e possibilita aos docentes, entre outros benefícios, realizar um acompanhamento individualizado.

Nesta perspectiva, estudos recentes publicados na literatura internacional mostram o impacto positivo do uso de STI em diferentes níveis e domínios educacionais [Steenbergen-Hu e Cooper 2014; VanLehn 2011; Ma et al. 2014], entretanto, segundo [González et al. 2014] enquanto os STI têm mostrado melhorar o desempenho dos estudantes, eles ainda têm grandes problemas em sua utilização e necessita de mais investigações empíricas. Para [Andrade et al. 2013; González et al. 2014] um dos principais problemas encontrados é o uso inadequado por parte dos estudantes, talvez devido ao tédio, falta de motivação e monotonia, que entre outros fatores causa comportamento incorreto do estudante ao interagir com o sistema. Para minimizar problemas dessa natureza, vários pesquisadores propõe implementar elementos de gamificação para aumentar o engajamento e promover a aprendizagem [Kapp 2012; Deterding et al. 2011]. O termo gamificação (do inglês: gamification) segundo [Kapp 2012] faz referência ao uso de mecânicas, estéticas e pensamento dos jogos para engajar pessoas, motivar a ação, promover a aprendizagem e resolver problemas.

No entanto, apesar do crescente interesse da comunidade científica na realização de estudos que apresente o impacto na aprendizagem dos estudantes com o uso de STI, ainda existem poucos trabalhos empíricos do uso de STI gamificado no contexto da Educação Básica no Brasil. Além disso, fica evidente a necessidade de realizar mais

\footnotetext{
${ }^{1}$ Programa Internacional de Avaliação de Estudantes (em inglês: Programme for International Student Assessment - PISA).
} 
VII Congresso Brasileiro de Informática na Educação (CBIE 2018)

Anais do XXIX Simpósio Brasileiro de Informática na Educação (SBIE 2018)

estudos que mostre evidências empíricas de práticas pedagógicas em cenário do mundo real, de modo a contribuir para desenvolvimento, uso e avaliação desse tipo de tecnologia no contexto educacional. Este artigo tem como objetivo extrair boas e más práticas pedagógicas a partir de processos de ensino e de aprendizagem usando um STI gamificado no Ensino Fundamental. Para tanto, realizou-se um estudo empírico misto com professores e estudantes, no domínio de Língua Portuguesa e Matemática. Os principais resultados mostram a extração de 10 boas práticas e 9 más práticas pedagógicas e observou-se melhorias significativas na aprendizagem dos estudantes com o uso de STI gamificado (denominado MeuTutor) no contexto da Educação Básica.

\section{Plataforma MeuTutor}

O MeuTutor ${ }^{2}$ é um STI gamificado, desenvolvido pela empresa MeuTutor Soluções Educacionais, uma startup brasileira criada dentro da Universidade Federal de Alagoas (UFAL), por pesquisadores de várias áreas do conhecimento, como: gestão, educação, marketing, design e tecnologia. É um ambiente educacional web, proprietário, que oferece suporte ao estudante de forma personalizada e gamificada [de Santana et al. 2016]. Os elementos de jogos presente no sistema são: pontos, troféus, rankings, barra de progresso, níveis, medalhas e avatares. Além disso, utiliza, entre outras técnicas, Bayesian Knowledge Tracing (BKT) [Yudelson et al. 2013] e Teoria de Resposta ao Item (TRI) [Couto e Primi 2011] para avaliar o conhecimento dos estudantes com base em seu sucesso e fracasso na resolução de problemas, bem como nas interações com os recursos de aprendizagem na plataforma. A partir das respostas, o sistema avalia quais tópicos de um tema específico precisa de reforço, e recomenda os recursos (questões e vídeos) para auxiliar na resolução das questões (aprendizagem adaptativa). A aprendizagem adaptativa visa aplicar métodos, técnicas e tecnologias, com objetivo de identificar as limitações e necessidades específicas de cada estudante ou grupo de estudantes e oferecer experiências de aprendizagem personalizada [Johnson et al. 2015].

\section{Trabalhos Relacionados}

Estudos recentes relacionados à utilização de ambientes gamificados para aumentar o engajamento e motivação dos estudantes tem sido conduzidos em vários domínios e níveis educacionais. Neste contexto, [De-Marcos et al. 2014] apresentam uma avaliação empírica comparando em um ambiente educacional online chamado BlackBoard, três abordagens: (1) gamificada, (2) rede social e (3) tradicional, com estudantes de um curso de graduação. Com objetivo de comparar as duas abordagens: gamificação e rede social, com relação a tradicional, no mesmo ambiente educacional. Para isso, foi realizado um estudo do tipo quase-experimental com pré-teste, pós-teste e questionário com 371 estudantes. Como resultados, no pré-teste não foi identificado diferença significativa entre os grupos experimentais e controle. No entanto, as análises do pósteste mostrou que ambos os grupos experimentais superaram o grupo de controle. Mas, surpreendentemente, os estudantes do grupo de controle superaram ambos dos grupos experimentais no exame escrito final.

No trabalho de [Hanus e Fox 2015] realizaram um estudo em sala de aula com objetivo de testar os efeitos da gamificação aplicada no contexto educacional, em cenário do mundo real. Para isso, foram selecionados 71 estudantes de duas turmas

\footnotetext{
${ }^{2}$ Disponível em: http://meututor.com.br/
} 
VII Congresso Brasileiro de Informática na Educação (CBIE 2018)

Anais do XXIX Simpósio Brasileiro de Informática na Educação (SBIE 2018)

diferentes do curso de comunicação. As aulas foram divididas em um currículo gamificado e outro não gamificado. Os principais resultados sugerem que algumas mecânicas comuns usadas na gamificação em sala de aula podem prejudicar os resultados educacionais. Além disso, a combinação de leaderboards, badges e mecânica de competição não melhoraram os resultados de aprendizagem e na pior das hipóteses pode prejudicar a motivação, satisfação e capacidade de aprender, mesmo a mecânica de jogos estando alinhada com os objetivos de aprendizagem.

Mais recentemente, [da Rocha Seixas et al. 2016] realizaram uma avaliação da eficácia de plataformas gamificadas como uma estratégia para o engajamento de estudantes do $8^{\circ}$ ano do Ensino Fundamental da rede pública de ensino no Brasil. Com objetivo de avaliar a mecânica de jogos através de gamificação para promover o engajamento de estudantes do Ensino Fundamental. Para isso, foram utilizadas duas plataformas web: ClassDojo e ClassBadges com 61 estudantes com idade entre 13 e 14 anos, divididos em dois grupos. Neste estudo, os pesquisadores utilizaram diferentes técnicas de pesquisa qualitativa exploratória e métodos quantitativos (observação, entrevista semiestruturada e questionários). Como resultados, observou-se que os estudantes demostraram curiosidade sobre as ferramentas e maior motivação. No geral, a investigação mostrou que gamificação teve efeitos positivos sobre o envolvimento dos estudantes no processo de aprendizagem.

Contudo, apesar dos estudos avaliarem empiricamente o impacto na aprendizagem, níveis de participação, engajamento, motivação e atitudes dos estudantes, comparando a eficácia dos elementos de gamificação e rede social com relação ao tradicional [De-Marcos et al. 2014] foi utilizado para isso, um Learning Management System (LMS), que não possui elementos de gamificação e componentes de rede social nativo. Vale ressaltar, também, que a plataforma não oferece feedback imediato e não faz recomendações de conteúdos para reforçar a aprendizagem com base no nível de dificuldade de cada estudante. Além disso, [Hanus e Fox 2015; da Rocha Seixas et al. 2016] não utilizaram STI gamificado, apesar dos pesquisadores reconhecerem que essas mecânicas de jogos são melhores se utilizadas através de tecnologias adaptativas, que provê feedback instantâneo, de modo a tornar o processo de aprendizagem personalizado.

\section{Materiais e Métodos}

Nesta seção, descreveremos os materiais e métodos aplicados no planejamento e execução do experimento controlado.

\subsection{Design do Experimento}

O presente estudo com design de experimento controlado [Montgomery 2013] foi realizado no município de São Sebastião, localizado na região agreste do Estado de Alagoas, com aplicação de pré-teste e pós-teste, com 191 estudantes e 12 professores da Educação Básica de 4 escolas da rede pública de ensino municipal. Neste estudo, os estudantes foram divididos em dois grupos: $(i)$ experimental e (ii) controle. No grupo experimental, os estudantes e professores utilizaram um STI gamificado (chamado MeuTutor) durante o período de abril a dezembro de 2015 (9 meses), enquanto que, os participantes do grupo de controle não utilizaram nenhuma tecnologia adaptativa durante o mesmo período para auxiliar nos processos de ensino e aprendizagem em Língua Portuguesa e Matemática. 
VII Congresso Brasileiro de Informática na Educação (CBIE 2018)

Anais do XXIX Simpósio Brasileiro de Informática na Educação (SBIE 2018)

\subsection{Participantes}

Participaram $N=191$ estudantes, 104 (54.45\%) são do gênero feminino e 87 (45.55\%) masculino, com idade entre 9 e 21 anos (média $=13.8$ e um desvio padrão $=2.01$ ). Desse total, participaram do grupo experimental $N=112$ estudantes, com faixa etária entre 9 e 21 anos (média $=14.44$ e um desvio padrão $=1.55)$, sendo $49(43.75 \%)$ masculino e $63(56.25 \%)$ feminino. Enquanto que, no grupo de controle participaram $N$ $=79$ estudantes, com faixa etária entre 10 e 18 anos (média $=12.90$ e um desvio padrão $=2.25)$, sendo $38(48.10 \%)$ do gênero masculino e $41(51.90 \%)$ feminino.

\subsection{Unidades Experimentais}

As unidades experimentais foram 4 (quatro) escolas de Educação Básica. De cada unidade escolar, foram selecionadas aleatoriamente 2 turmas, sendo, uma turma do $5^{\circ}$ ano (ano final do ensino fundamental I) e outra do $9^{\circ}$ ano (ano final do ensino fundamental II), totalizando 8 turmas, ou seja, 4 turmas de cada etapa dos anos finais do Ensino Fundamental I e II.

\subsection{Instrumentação}

A priori, foi aplicado um pré-teste com objetivo de diagnosticar as habilidades dos estudantes em Língua Portuguesa e Matemática, para ambas os grupos. Após isso, os estudantes e professores do grupo experimental tiveram acesso ao STI gamificado (plataforma MeuTutor), como ferramenta para auxiliar nos processos de ensino e de aprendizagem. No final do experimento foi aplicado um pós-teste. Além disso, durante o período de execução do experimento foram feitas anotações em um diário de bordo do pesquisador com as observações in loco, fotos, informações descritivas, as falas, comportamento dos estudantes e professores no laboratório de informática. De acordo com [Lüdke e André 2013] essas medidas têm um caráter meramente prático, no sentido de ajudar a organização e análise qualitativa dos dados. Por último, foi utilizado o software $\mathrm{R}^{3}$, para realizar análise estatística. Dessa forma, o presente estudo caracterizase como pesquisa mista. Segundo [Creswell 2010] métodos mistos é uma abordagem de investigação que combina os métodos qualitativo e quantitativo.

O pré-teste e pós-teste foram elaborados com base nas matrizes de referência e descritores da "Prova Brasil" desenvolvido pelo MEC, e refere-se às competências mínimas esperadas que o estudante tenha aprendido no que diz respeito ao desenvolvimento cognitivo em Língua Portuguesa e Matemática, em cada etapa do Ensino Fundamental. Para os estudantes do $5^{\circ}$ ano, o pré-teste foi composto por 4 blocos com 11 questões objetivas de multiplica escolha em cada bloco ( 2 blocos com questões de Língua Portuguesa e outros 2 com questões de Matemática). Para os estudantes do $9^{\circ}$ ano, o pré-teste foi composto pelas mesmas disciplinas e quantidade de blocos, no entanto, cada bloco foi elaborado com 13 questões. O pós-teste foi elaborado com o mesmo número de questões e formato do pré-teste.

\subsection{Coleta e Análise dos Dados}

Os dados foram coletados a partir das observações in loco registradas no diário de bordo do pesquisador, da aplicação do pré-teste impresso, realizado no mês de março de 2015 ,

\footnotetext{
${ }^{3}$ Disponível em: https://www.r-project.org/
} 
VII Congresso Brasileiro de Informática na Educação (CBIE 2018)

Anais do XXIX Simpósio Brasileiro de Informática na Educação (SBIE 2018)

pós-teste impresso, aplicado logo após o término do experimento no mês de dezembro de 2015. Em seguida, foram coletados os dados dos estudantes do grupo experimental, referente aos elementos de gamificação (nível, pontos, troféus e ranking), presentes na Plataforma MeuTutor e proveniente das interações dos estudantes com a plataforma. Além disso, foram observadas as intervenções dos professores com o uso do STI gamificado nos processos de ensino-aprendizagem.

A análise quantitativa envolveu o tratamento dos dados proveniente do pré-teste e pós-teste. Nesta etapa, foram excluídos os estudantes que ingressaram nos grupos de controle e experimental após a aplicação do pré-teste. A análise estatística foi realizada através dos modelos de regressão beta e análises de diagnóstico para verificar se um determinado modelo é uma representação adequada dos dados [Ferrari e Cribari-Neto 2004; Espinheira et al. 2008; Espinheira et al. 2015]. Os testes foram realizados com nível de confiança de $90 \%$ e $95 \%$, considerando um $\propto=0.1$ e $\propto=0.05$. Além disso, foi aplicado o Framework Analysis como abordagem de análise qualitativa [Spencer e Ritchie 2002]. Essa abordagem envolve um processo sistemático de filtragem, mapeamento e classificação do material de acordo com as principais questões e temas. Na próxima seção, apresentamos os resultados e discussão extraídos no presente estudo empírico.

\section{Resultados e Discussão}

Nesta seção, apresentaremos os resultados com base nos métodos de pesquisa quantitativa e qualitativa. Entretanto, vale ressaltar que a aplicação da análise qualitativa é de fundamental importância para compreender melhor o cenário investigado e para complementar os resultados apresentados através da abordagem quantitativa. Nos próximos parágrafos, descreveremos as Boas Práticas (BP) e as Más Práticas (MP) pedagógicas a partir de processos de ensino e de aprendizagem usando um STI gamificado, extraídas com base nas constatações das análises.

BP1 - Aplicar STI gamificado no processo de ensino-aprendizagem em Língua Portuguesa e Matemática. Com 5\% de significância, constatou-se que não existe diferença significativa entre os gêneros feminino e masculino quanto ao desempenho em Língua Portuguesa ( $p$-value $=0.510)$. Enquanto que, em Matemática, os estudantes masculinos obtiveram um desempenho de $18.13 \%$ superior em relação ao feminino $(p$-value $=0.012)$. O uso do STI gamificado apresentou evidências de melhoria em Língua Portuguesa para ambos os gêneros, com aumento de $49.18 \%$ para masculino e $22.14 \%$ feminino. Em Matemática, o desempenho foi de $24.61 \%$ e $19.72 \%$, respectivamente. Além disso, constatou-se um aumento de $23.37 \%$ e $34.99 \%$ do gênero masculino onde as famílias tem o menor indicador de nível socioeconômico ( $p-$ Value $\leq 0.1)$. O mesmo foi observado para os estudantes residentes tanto na área urbana quanto na zona rural $(p$-value $=0.005)$ e $(p$-value $=0.000)$, respectivamente.

BP2 - Realizar atividade colaborativa com o uso de STI gamificado. Através da análise qualitativa observou-se que os estudantes do grupo experimental utilizaram o STI gamificado, em média 2 vezes por semana, com duração de 1 hora/aula cada, no laboratório de informática. Além disso, notou-se que alguns estudantes com habilidades já adquiridas em determinados assuntos, atuaram como tutores voluntários auxiliando outros colegas na resolução de problemas através da plataforma. BP3 - Configurar planos de aulas para reforçar a aprendizagem dos estudantes nos domínios de 
VII Congresso Brasileiro de Informática na Educação (CBIE 2018)

Anais do XXIX Simpósio Brasileiro de Informática na Educação (SBIE 2018)

Língua Portuguesa e Matemática. Observou-se que o STI gamificado foi utilizado pelos professores para configurar planos com conteúdos específicos (questões e vídeos) para reforçar a aprendizagem dos estudantes. BP4 - Elaborar simulados para diagnosticar as habilidades cognitivas dos estudantes. Os professores utilizaram o STI gamificado para elaborar simulados, como estratégias para verificar as habilidades cognitivas dos estudantes nos domínios de Língua Portuguesa e Matemática.

BP5 - Criar exercícios de reforço nos domínios de Língua Portuguesa e Matemática. Os professores utilizaram o STI gamificado para criar exercícios de forma automática e/ou personalizada com o objetivo de reforçar a aprendizagem dos estudantes em domínios específicos do conhecimento. BP6 - Utilizar o elemento de gamificação "troféu". Com um nível de significância de $p-$ Value $\leq 0.1$, observouse que, a cada 1 troféu conquistado pelo estudante, aumenta o seu desempenho em $2.1 \%$ no domínio de Língua Portuguesa e 3.0\% em Matemática. BP7 - Mostrar o elemento de gamificação "nível" do estudante dentro do STI gamificado. Além do elemento de gamificação "troféu", o "nível" mostrou-se relevante para o desempenho em Matemática $(p$-value $=0.016)$. Constatou-se que, quanto maior o nível do estudante dentro do STI gamificado, melhor foi o seu desempenho em Matemática.

BP8 - Realizar exercícios e/ou simulados no laboratório de informática. Os professores interagiram com o STI gamificado para acompanhar relatórios que mostram os pontos fortes e fracos da turma e por estudante, individualmente, para verificar o número de questões respondidas corretas e incorretas. BP9 - Fazer atividade para casa com o uso de STI gamificado. Observou-se que os professores utilizaram a plataforma para criar exercícios de forma automática e/ou personalizada visando reforçar a aprendizagem dos estudantes. Além disso, evidenciou-se melhoria no desempenho com o uso da plataforma para realizar exercícios como atividade de casa. BP10 - Promover o engajamento dos estudantes com o uso do STI gamificado. Notou-se que alguns professores criaram exercícios e simulados de Língua Portuguesa e Matemática, para os estudantes resolverem com o uso da plataforma. Além disso, para manter os estudantes ainda mais engajados, os professores atribuíram pontos extras para complementar uma avaliação presencial.

MP1 - Avaliar o desempenho do estudante exclusivamente com o uso do STI gamificado. Percebe-se que ao realizar as atividades em equipe, os estudantes adquirem novas habilidades sociais, além de reforçar a aprendizagem. Com base nessas evidências, nota-se que a avaliação exclusiva com o uso da plataforma é limitada no contexto da educação básica. MP2 - Propor o uso de STI gamificado com o mesmo design de gamificação para estudantes dos gêneros feminino e masculino. Com $5 \%$ de significância, os estudantes do gênero masculino obtiveram um desempenho de $18.13 \%$ superior ao feminino $(p$-value $=0.012)$. Com $p-$ Value $\leq 0.1$, o uso de STI gamificado apresentou evidências de melhoria em Língua Portuguesa para ambos os gêneros. Entretanto, os estudantes do gênero feminino obtiveram resultados inferiores com relação ao masculino, em ambas as disciplinas. [Albuquerque et al. 2017] confirma que o gênero é um fator que impacta diretamente no design de gamificação.

MP3 - Utilizar STI gamificado com estudantes do $5^{\circ}$ ano ainda em processo de alfabetização. Observou-se que os professores e estudantes do $9^{\circ}$ ano utilizaram mais a plataforma do que os do $5^{\circ}$ ano, isso porque, muitos estudantes do $5^{\circ}$ ano, ainda estão em processo de alfabetização e encontram dificuldades em leitura e interpretação 
VII Congresso Brasileiro de Informática na Educação (CBIE 2018)

Anais do XXIX Simpósio Brasileiro de Informática na Educação (SBIE 2018)

de textos e na resolução de problemas. Neste estudo, STI gamificado tinha como objetivo reforçar a aprendizagem e não de alfabetizá-los. MP4 - Utilizar os recursos do STI gamificado não alinhado com a proposta pedagógica. Apesar dos professores conhecerem a plataforma e os recursos disponíveis, observou-se que, alguns não utilizaram corretamente, ou seja, a falta de planejamento para o uso adequada da tecnologia deixou os estudantes entediados, desmotivados e desengajados para avançar no processo de aprendizagem. MP5 - Usar o STI gamificado sem preparar a conectividade da escola. A baixa conexão da Internet nas escolas deixou os professores e estudantes entediados. Devido a isso, reduziu-se a interação com o STI gamificado no laboratório de informática.

MP6 - Utilizar o elemento de gamificação "pontos". Com um nível de significância de 5\%, o elemento de gamificação "pontos" presente no STI gamificado não apresentou evidências de melhoria na aprendizagem ( $p$-value $=0.976)$. MP7 Mostrar o elemento "ranking" dentro do STI gamificado. O elemento de gamificação "ranking" na plataforma não mostrou melhoria no desempenho dos estudantes $(p$-value $=0.889)$. MP8 - Limitar a conexão à Internet dentro da escola para acesso ao STI gamificado. Na escola, apesar de existir conexão Wi-Fi, os estudantes não tinham permissão para utilizar no ambiente escolar. $O$ acesso foi limitado para uso apenas no laboratório de informática, em dias e horários previamente agendados pelo professor ou estudante. MP9 - Deixar os alunos interagirem livremente com o STI gamificado no laboratório de informática. Observou-se que em alguns momentos os estudantes interagiram livremente com o STI gamificado, ou seja, sem a presença do professor. Diante disso, alguns estudantes utilizaram para navegar na web, acessar sites de entretenimento e redes sociais.

\section{Considerações Finais}

Os resultados mais relevantes mostram: $i$ ) melhoria na aprendizagem dos estudantes com o uso do STI gamificado, em Língua Portuguesa e Matemática; ii) estudantes do gênero masculino obtiveram um desempenho em Matemática de $18.13 \%$ superior ao gênero feminino; iii) uso da plataforma MeuTutor pelos estudantes do gênero masculino apresentou um aumento no desempenho de $49.18 \%$, contra $22.14 \%$ em relação ao gênero feminino no domínio de Língua Portuguesa; iv) os elementos de gamificação "nível" e "troféu" evidenciou um aumento no desempenho de $2.1 \%$ em Língua Portuguesa e 3.0\% no domínio de Matemática. Porém, os elementos de gamificação "pontos" e "ranking" não apresentaram evidências significantes; v) extração de 10 boas práticas e 9 más práticas pedagógicas com o uso de um STI gamificado no contexto da Educação Básica, extraídas com base nas constatações observadas das análises quantitativa e qualitativa.

Com base nessas evidências, propomos quatro recomendações para construção de STI gamificado: (i) análise da maturidade tecnológica - essencial fazer uma análise da maturidade tecnológica da escola para personalização do STI gamificado considerando as informações de background e coletar informações contextuais sobre o perfil do estudante, localização, período de realização das atividades e tipo de dispositivo utilizado; (ii) Design de gamificação - módulo responsável pela personalização em tempo real considerando o perfil do estudante; (iii) autoria da tutoria e da gamificação - permitir ao professor criar seu próprio conteúdo, selecionar emblemas e associar um valor personalizado como forma de recompensa; e (iv) learning 
VII Congresso Brasileiro de Informática na Educação (CBIE 2018)

Anais do XXIX Simpósio Brasileiro de Informática na Educação (SBIE 2018)

analytics - módulo de visualização de dados que oferece ao professor relatórios para descobrir padrões de aprendizagem individuais e em grupo.

Contudo, conclui-se que o uso adequado de STI gamificado como ferramenta complementar para auxiliar docentes e estudantes nos processos de ensinoaprendizagem contribui significativamente para melhoria do desempenho em domínios específicos do conhecimento. Como trabalhos futuros, pretende-se realizar um experimento controlado longitudinal para validar as boas e más práticas pedagógicas extraídas com o uso da plataforma em escolas públicas localizadas geograficamente em regiões diferentes no cenário brasileiro. Além disso, investigar o perfil dos professores da Educação Básica e qual a sua influência no desempenho dos estudantes com a utilização de tecnologias educacionais de aprendizagem adaptativa gamificada.

\section{Referências}

Albuquerque, J., Bittencourt, I. I., Coelho, J. A., \& Silva, A. P. (2017). Does gender stereotype threat in gamified educational environments cause anxiety? An experimental study. Computers \& Education, 115, 161-170.

Andrade, F. R., Pedro, L. Z., Lopes, A. M. Z., Bittencourt, I. I., \& Isotani, S. (2013). Desafio do Uso de Gamificação em Sistemas Tutores Inteligentes Baseados em Web Semântica. In XXXIII Congresso da Sociedade Brasileira de Computação (Vol. 1, pp. 1453-1462).

Barbosa, A. F. (2016). Pesquisa sobre o uso das tecnologias de informação e comunicação nas escolas brasileiras: tic educação 2015. São Paulo: Comitê Gestor da Internet no Brasil.

Brasil. (2016). Brasil no PISA 2015: análise e reflexões sobre o desempenho dos estudantes brasileiros. São Paulo: Fundação Santillana.

Brasil. Instituto Nacional de Estudos e Pesquisas Educacionais Anísio Teixeira. (2018). Relatório do $2^{\circ}$ Ciclo de Monitoramento das Metas do Plano Nacional de Educação 2018. Brasília, DF: INEP.

Couto, G., \& Primi, R. (2011). Teoria de resposta ao item (TRI): conceitos elementares dos modelos para itens dicotômicos. Boletim de Psicologia, 61(134), 1-15.

Creswell, John W. (2010). Projeto de Pesquisa: métodos qualitativo, quantitativo e misto. $3^{\mathrm{a}}$ ed. - Porto Alegre: Artmed.

da Rocha Seixas, L., Gomes, A. S., \& de Melo Filho, I. J. (2016). Effectiveness of gamification in the engagement of students. Computers in Human Behavior, 58, 4863.

de Santana, S. J., Paiva, R., Bittencourt, I. I., Ospina, P. E., de Amorim Silva, R., \& Isotani, S. (2016a). Evaluating the impact of Mars and Venus Effect on the use of an Adaptive Learning Technology for Portuguese and Mathematics. In Advanced Learning Technologies (ICALT), 2016 IEEE 16th International Conference on (pp. 31-35). IEEE.

De-Marcos, L., Domínguez, A., Saenz-de-Navarrete, J., \& Pagés, C. (2014). An empirical study comparing gamification and social networking on elearning. Computers \& Education, 75, 82-91. 
VII Congresso Brasileiro de Informática na Educação (CBIE 2018)

Anais do XXIX Simpósio Brasileiro de Informática na Educação (SBIE 2018)

Deterding, S., Dixon, D., Khaled, R., \& Nacke, L. (2011). From game design elements to gamefulness: defining gamification. In Proceedings of the 15th international academic MindTrek conference: Envisioning future media environments (pp. 9-15). ACM.

Espinheira, P. L., da Silva, L. C. M., \& Silva, A. D. O. (2015). Prediction Measures in Beta Regression Models. arXiv preprint arXiv:1501.04830.

Espinheira, P. L., Ferrari, S. L., \& Cribari-Neto, F. (2008). On beta regression residuals. Journal of Applied Statistics, 35(4), 407-419.

Ferrari, S., \& Cribari-Neto, F. (2004). Beta regression for modelling rates and proportions. Journal of Applied Statistics, 31(7), 799-815.

González, C., Mora, A., \& Toledo, P. (2014). Gamification in intelligent tutoring systems. In Proceedings of the Second International Conference on Technological Ecosystems for Enhancing Multiculturality (pp. 221-225). ACM.

Hanus, M. D., \& Fox, J. (2015). Assessing the effects of gamification in the classroom: A longitudinal study on intrinsic motivation, social comparison, satisfaction, effort, and academic performance. Computers \& Education, 80, 152-161.

Johnson, L., Becker, S. A., Estrada, V., \& Freeman, A. (2015). NMC horizon report: 2015 K (Vol. 6101). New Media Consortium.

Kapp, K. M. (2012). The gamification of learning and instruction: game-based methods and strategies for training and education. John Wiley \& Sons.

Lüdke, Menga; André, Marli E. D. A. (2013). Pesquisa em educação: abordagens qualitativas. $2^{\mathrm{a}}$ ed. - Rio de Janeiro: E.P.U.

Ma, W., Adesope, O. O., Nesbit, J. C., \& Liu, Q. (2014). Intelligent tutoring systems and learning outcomes: A meta-analysis. Journal of Educational Psychology, 106(4), 901.

Montgomery, D. C. (2013). Design and analysis of experiments. John wiley \& sons.

OECD. (2016). PISA 2015 Results (Volume I): Excellence and Equity in Education, PISA, OECD Publishing, Paris.

QEdu. (2018). Portal QEdu. Disponível em: http://qedu.org.br/

Spencer, L., \& Ritchie, J. (2002). Qualitative data analysis for applied policy research. In Analyzing qualitative data (pp. 187-208). Routledge.

Steenbergen-Hu, S., \& Cooper, H. (2014). A meta-analysis of the effectiveness of intelligent tutoring systems on college students' academic learning. Journal of Educational Psychology, 106(2), 331.

VanLehn, K. (2011). The relative effectiveness of human tutoring, intelligent tutoring systems, and other tutoring systems. Educational Psychologist, 46(4), 197-221.

Yudelson, M. V., Koedinger, K. R., \& Gordon, G. J. (2013). Individualized bayesian knowledge tracing models. In International Conference on Artificial Intelligence in Education (pp. 171-180). Springer, Berlin, Heidelberg. 